\title{
Determination the Place of Power System Reactive Compensation by Modal Analysis Method
}

\author{
Qinghai Zhu ${ }^{1, \text { a }}$, Shunxiang $\mathrm{Cai}^{1}$, Tao Xu ${ }^{2}$, Dongfang Zhang ${ }^{2, \mathrm{~b}}$ \\ ${ }^{1}$ Liupanshui Power Supply Bureau, Guizhou Power Grid Corporation, Liupanshui, Guizhou Province \\ China \\ ${ }^{2}$ the College of Electrical Engineering, Guizhou University, Guiyang, 550025, Guizhou Province, \\ China \\ a245982238@qq.com \\ b396048901@qq.com
}

Keywords: Power System, Modal Analysis, Reactive Power Compensation, Participation Factor, Steady-state stability.

\begin{abstract}
Determination of the location where the shunt reactive power compensation devices is important for the enhancement of the power system voltage stability and the decreasing of power losses. With the method of modal analysis, this paper adopts bus voltage participation factors to solve this problem. In order to show the effectiveness of the proposed method, the New England 39-bus power system with shunt reactive power compensation devices is used as an example. Test results show that with the reactive power compensators located at the place which the proposed method determines, the voltage stability is considerably increased.
\end{abstract}

\section{Introduction}

The shunt var compensation at suitable location improves the power system voltage stability to a great extent [1-2]. Lots of researches using linearzing method to analyze this problem, which gets wonderful results to the power system characterized with linear performance. The modal analysis was adopted in [3] to determine the suitable location of SVC for purpose of the voltage stability enhancement. In order to improve the steady-state security, the index of V-Q sensitivity was adopted to allocate the shunt compensator in [4]. In [5] the right eigenvector was studied to optimize the allocation of SVC. Reference [6] and [7] extended the traditional modal analysis method and proposed active power participation factor to study the effect of generator active power output to the transmission capability. However, because of the conditions of economy, environment and the society etc, power systems operating economically is more focused. In this paper, the modal analysis is adopted to study the allocation of shunt var compensation for the purpose of reducing power losses. The New England 39-bus system is studied, and the simulation results show the efficiency of the method.

\section{Power flow equations and its linear transformation}

Voltage stability is a subset of overall power system stability. It can be investigated by the use of the power flow equations. In this paper, the power system is modeled by the following power flow equations.

$$
\begin{aligned}
& P_{i}=V_{i} \sum_{j=1}^{n} V_{j}\left(G_{i j} \cos \theta_{i j}+B_{i j} \sin \theta_{i j}\right) \quad i=1,2 . . n-1 \\
& Q_{i}=V_{i} \sum_{j=1}^{n} V_{j}\left(G_{i j} \sin \theta_{i j}-B_{i j} \cos \theta_{i j}\right) \quad i=1,2 . . m
\end{aligned}
$$

where $V_{i}$ is the voltage at bus $i, \theta_{i j}$ is the angle difference between buses $i$ and $j, G_{i j}$ and $B_{i j}$ are the conductance and susceptance of the line connecting the buses $i$ and $j$ respectively, $P_{i}$ and $Q_{i}$ are the 
active and the reactive power injections at bus $i$ respectively, and $m$ and $n$ are the number of PQ buses and total buses of the system respectively.

For the sake of simplification, the equation (1) can be rewritten as following.

$$
L=g(x)
$$

where $L=\left(P_{1}, P_{2}, \ldots, P_{n-1}, Q_{1}, Q_{2}, \ldots, Q_{m}\right)^{T}, \boldsymbol{x}=\left(\theta_{1}, \theta_{2}, \ldots, \theta_{n-1}, V_{1}, V_{2}, \ldots, V_{m}\right)^{T}$.

The Taylor's series expansion of equation (2) about an equilibrium point (EP) is given by Eq. (3).

$$
L=\boldsymbol{J} \boldsymbol{x}+H \cdot O \cdot T
$$

where $\boldsymbol{J}$ is the Jacobian matrix, and H.O.T is the high order terms.

Using the similarity transformation $\boldsymbol{x}=\boldsymbol{W} \boldsymbol{y}$, where $\boldsymbol{W}$ is the matrix of right eigenvectors for $\boldsymbol{J}$, the original system can be represented by the following Taylor expansion with up to first order in the $y$-coordinates.

$$
\boldsymbol{W}^{-1} \mathbf{L}=\Lambda y
$$

where $\Lambda$ is the eigenvalue matrix of $\boldsymbol{J}$.

\section{Participation factor (PF)}

The bus voltage participation factors provide useful information about the power system performance. The bus voltage participation factors with the interesting mode give the degree of contribution of the buses to the corresponding system. The larger the bus voltage participation factor is, the more influence the bus will contribute to the voltage stability. Consequently, this bus is the appropriate location where var compensation should be used for the voltage stability enhancement of the overall power system. In this paper, the bus voltage participation factor is used as an index to measure this kind of contribution and it can be computed by Eq. (5).

$$
p_{k i}=w_{k i} \times v_{i k}
$$

where $w_{k i}$ and $v_{i k}$ are the element of $\boldsymbol{W}$ and $\boldsymbol{W}^{1}$ respectively. $p_{k i}$ represents a measure of the participation of the $k$ th state to the $i$ th mode.

\section{Ranking buses}

As has been mentioned in the previous section, based on the power flow equations, this paper uses the bus voltage participation factor $p_{k i}$ as an index to measure the var compensation allocation. The large the bus voltage participation factor associated with the interesting mode indicates more impact of the bus on the voltage stability. Consequently, it is also the most effective installation location of var compensation.

The results of reference [9] pointed out that the eigenvectors of the full Jacobian matrix are always equal to those of the reduced Jacobian matrix and the information of the full Jacobian matrix eigenvectors is all included in the eigenvectors of the reduced Jacobian matrix at the singularity point. However, at the non-singularity points, the eigenvector of the full Jacobian matrix do not match those of the reduced Jacobian matrix. Furthermore, the larger the operating condition is away from the singularity point, the larger the difference between the eigenvectors is. The conclusion that the reduced matrices contain all information of the full Jacobian is only valid at the singularity point. Therefore, in order to get correct information, this paper analyzes the full power flow equations before the singularity point.

\section{Case study}

The effectiveness of the proposed method has been tested on the New England 39-bus test system 
shown in Fig 1. In order to study the effect of var compensation on the voltage stability enhancement and power losses reducing, the indices in Eq. (6) and Eq. (7) are used in this paper.

$$
\begin{array}{r}
V S E=\frac{\lambda_{\min }^{\text {Var }}-\lambda_{\min }}{\lambda_{\min }} \cdot 100 \% \\
P L R=\frac{P_{\text {loss }}-P_{\text {loss }}^{\text {Var }}}{P_{\text {loss }}} \cdot 100 \%
\end{array}
$$

where $\lambda_{\min }^{\mathrm{Var}}$ and $P_{\text {loss }}^{\mathrm{Var}}$ are the smallest eigenvalue and power losses after var compensation respectively, $\lambda_{\min }$ and $P_{\text {loss }}$ are the smallest eigenvalue and power losses before var compensation.

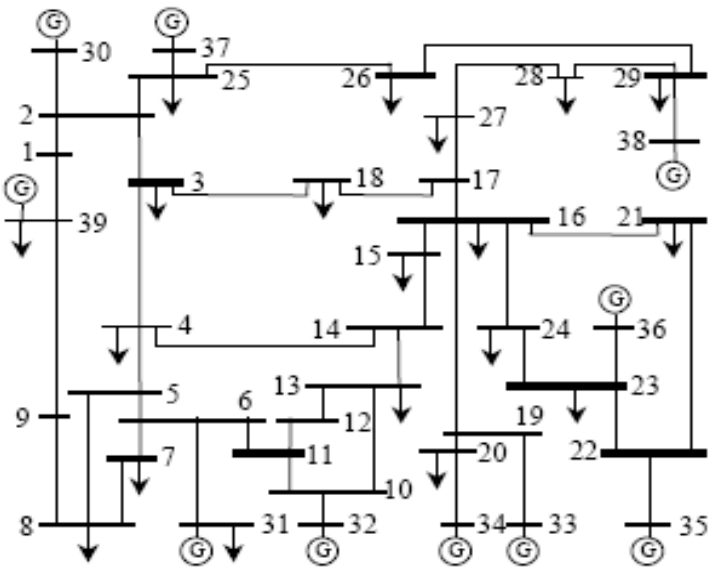

Fig. 1 New England 39-bus system

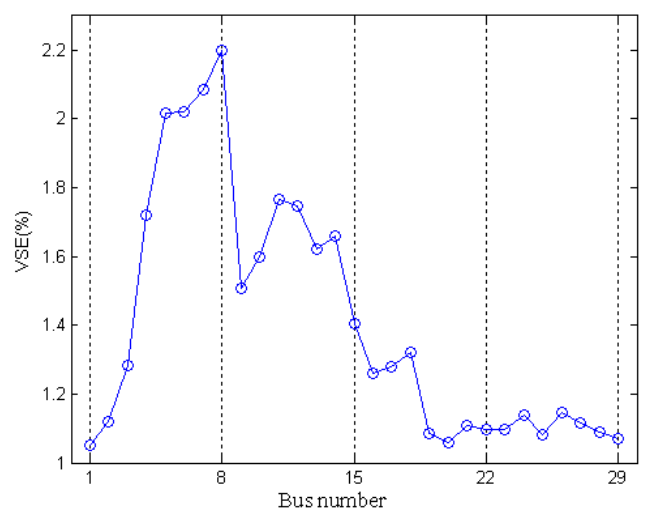

Fig. 3 VSE corresponding to var compensation at each bus

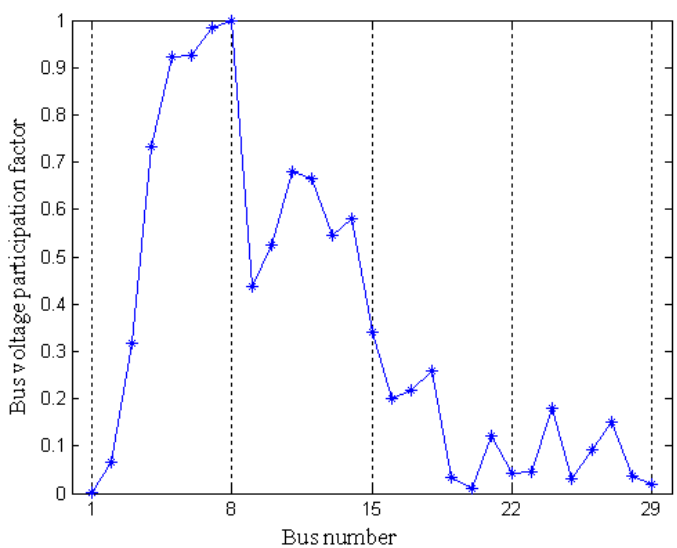

Fig.2 bus voltage participation factor corresponding to the interesting mode

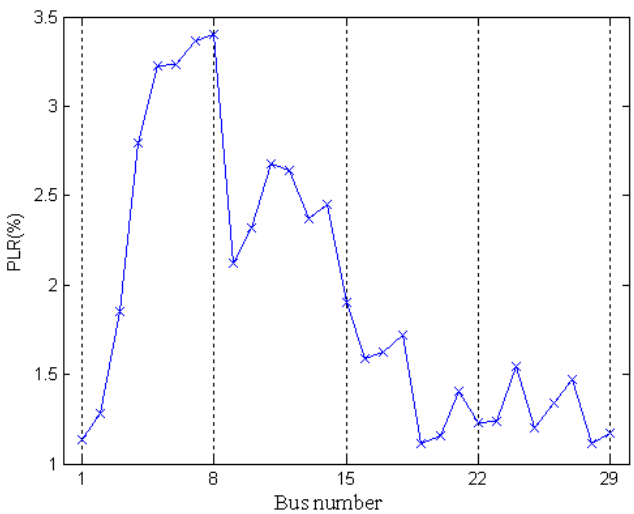

Fig. 4 PLR corresponding to var compensation at each bus

The mode corresponding to the smallest eigenvalue is considered as the interesting mode for the voltage stability. The bus voltage participation factors with the interesting mode are calculated and normalized with respect to the largest component respectively. The results are shown in Fig 2. From Fig. 2, the bus voltage participation factor corresponding to bus 8 is the largest one, which shows bus 8 is the suitable bus for var compensation to improve the voltage stability. In order to verify the effect of the method, var compensation at each bus is simulated and the indices of VSE are calculated and shown in Fig. 3. Comparing Fig. 2 and Fig. 3, it can be seen that compensation the bus of large bus voltage participation factor, the corresponding index of VSE is large. It shows that the method of modal analysis is effective to determine the location of var compensation for voltage stability.

Because either the reactive power or the voltage is relative to the power losses, the power losses reducing corresponding to var compensation at each bus is simulated and shown in Fig. 4. Comparing Fig. 2, Fig. 3 with Fig. 4, it can be seen that by using modal analysis to determine the location of var compensation, both the voltage stability enhancement and the power losses reducing are obtained 
simultaneously.

\section{Conclusions}

The method of modal analysis is adopted to study the power system power flow equations, and the bus voltage participate factor is used to determine the location of var compensation for both voltage stability enhancement and power losses reducing. The simulation results show that by using modal analysis to determine the location of var compensation, both the voltage stability enhancement and the power losses reducing are obtained simultaneously.

\section{Acknowledgments}

We would like to thank the project supported by Science and Technology Foundation of Guizhou Province([2009]2236). This work is also supported by the Talent Foundation of Guizhou University ( (2008)011), the Science and Technology Funds for Excellent Young Talents of Guizhou Province([2009]14).

\section{References}

[1] M. Moghavvemi, M. O. Faruque: TENCON2000, Vol.2 (2000), p. 357.

[2] N. Boonpirom, K. Paitoonwattanakij: The 7th International Power Engineering Conference, 2005, p. 1.

[3] Wang Jie, Ruan Yingqin, Fu Le, et al: Proceedings of the CSEE, Vol. 24 (2004), p. 24.

[4] B. Gao, G. K. Morison, P. Kundur: IEEE Transactions on Power Systems, Vol. 7 (1992), p. 1529.

[5] Y. Mansour, Wilsun Xu, F. Alvarado, et al: IEEE Transactions on Power Systems, Vol. 9 (1994), p. 757.

[6] Sung-Hwan Song, Jung-Uk Lim, Seung-II Moon: Electric Power Systems Research, Vol. 70 (2004), p. 7.

[7] F. Jurado, J. A. Rodriguez: Proceedings ETFA’99, Vol. 2 (1999), p. 1193.

[8] Zhang Jing, Wen Jin-yu, Cheng Shi-jie: Proceedings of the CSEE, Vol. 26 (2006), p. 1.

[9] L. C. P. da Silva, da Costa, W. Xu: IEEE Power Engineering Society Summer Meeting, Vol. 3 (2000), p. 1946. 